\title{
Microbial transmission from mothers with obesity or diabetes to infants: an innovative opportunity to interrupt a vicious cycle
}

\author{
Taylor K. Soderborg ${ }^{1}$ - Sarah J. Borengasser ${ }^{2}$ - Linda A. Barbour ${ }^{3,4}$. \\ Jacob E. Friedman ${ }^{1,3,5}$
}

Received: 13 October 2015 / Accepted: 6 January 2016/Published online: 2 February 2016

(C) Springer-Verlag Berlin Heidelberg 2016

\begin{abstract}
Maternal obesity and diabetes dramatically increase the long-term risk for obesity in the next generation, and pregnancy and lactation may be critical periods at which to aim primary prevention to break the obesity cycle. It is becoming increasingly clear that the gut microbiome in newborns and infants plays a significant role in gut health and therefore child development. Alteration of the early infant gut microbiome has been correlated with the development of childhood obesity and autoimmune conditions, including asthma, allergies and, more recently, type 1 diabetes. This is likely to be due to complex interactions between mode of delivery, antibiotic use, maternal diet, components of
\end{abstract}

Jacob E. Friedman

jed.friedman@ucdenver.edu

1 Department of Pediatrics, Section of Neonatology, University of Colorado School of Medicine, Anschutz Medical Campus, Mail Stop 8106, 12801 East 17th Avenue, Aurora, CO 80045, USA

2 Department of Pediatrics, Section of Nutrition, University of Colorado School of Medicine, Anschutz Medical Campus, Aurora, CO, USA

3 Department of Medicine, Division of Endocrinology, Metabolism, and Diabetes, University of Colorado School of Medicine, Anschutz Medical Campus, Aurora, CO, USA

4 Department of Obstetrics and Gynecology, Division of Maternal-Fetal Medicine, University of Colorado School of Medicine, Anschutz Medical Campus, Aurora, CO, USA

5 Department of Biochemistry and Molecular Genetics, University of Colorado School of Medicine, Anschutz Medical Campus, Aurora, CO, USA breastfeeding and a network of regulatory events involving both the innate and adaptive immune systems within the infant host. Each of these factors are critical for informing microbiome development and can affect immune signalling, toxin release and metabolic signals, including short-chain fatty acids and bile acids, that regulate appetite, metabolism and inflammation. In several randomised controlled trials, probiotics have been administered with the aim of targeting the microbiome during pregnancy to improve maternal and infant health but the findings have often been confounded by mode of delivery, antibiotic use, ethnicity, infant sex, maternal health and length of exposure. Understanding how nutritional exposure, including breast milk, affects the assembly and development of both maternal and infant microbial communities may help to identify targeted interventions during pregnancy and in infants born to mothers with obesity or diabetes to slow the transmission of obesity risk to the next generation. The aim of this review is to discuss influences on infant microbiota colonisation and the mechanism(s) underlying how alterations due to maternal obesity and diabetes may lead to increased risk of childhood obesity.

Keywords Diabetes · Infant · Maternal · Microbiome · Obesity $\cdot$ Pregnancy $\cdot$ Review

$\begin{array}{ll}\text { Abbreviations } \\ \text { BA } & \text { Bile acid } \\ \text { FXR } & \text { Farnesoid X receptor } \\ \text { GDM } & \text { Gestational diabetes mellitus } \\ \text { GPR } & \text { G protein-coupled receptor } \\ \text { GWG } & \text { Gestational weight gain }\end{array}$




$\begin{array}{ll}\text { HFD } & \text { High-fat diet } \\ \text { HMO } & \text { Human milk oligosaccharide } \\ \text { LGG } & \text { Lactobacillus rhamnosus strain GG } \\ \text { LPS } & \text { Lipopolysaccharide } \\ \text { NAFLD } & \text { Non-alcoholic fatty liver disease } \\ \text { NHP } & \text { Non-human primate } \\ \text { RCT } & \text { Randomised controlled trial } \\ \text { SCFA } & \text { Short-chain fatty acid } \\ \text { TGR5 } & \text { G protein-coupled BA receptor 1 } \\ \text { Treg } & \text { T regulatory } \\ \text { WSD } & \text { Western-style diet }\end{array}$

\section{Introduction: gestational diabetes and obesity affect the health of both mother and infant}

The developmental origins of health and disease (DoHAD) hypothesis, now substantiated by extensive research in animals and humans, suggests that both maternal nutrient deficiency and nutrient excess in utero and in early infancy programs a susceptibility to metabolic disease later in life [1-3]. The importance of minimising risks associated with maternal obesity and/or diabetes is no longer limited to improving the immediate pregnancy outcomes for mother and infant but, even more challenging, includes improving the long-term metabolic health of the affected offspring [4].

Maternal obesity and diabetes are consistently among the most powerful predictors of childhood obesity [5] and other adverse health conditions, such as type 2 diabetes, non-alcoholic fatty liver disease (NAFLD) and the metabolic syndrome, now emerging in children as young as 6 years of age [6]. Maternal Western-style diet (WSD) and insulin resistance in non-human primates (NHPs) promote lipotoxicity in the fetal liver [7] and NAFLDlike changes that persist in the offspring despite weaning to a healthy diet [8]. In human infants, using nuclear magnetic resonance spectroscopy, newborns from mothers with gestational diabetes mellitus (GDM) and obesity show evidence of increased intrahepatic fat at birth [9], potentially increasing the risk for NAFLD in adolescence [10]. Changes in behaviour, including appetite, anxiety and aggression, as well as neurological neurotransmitter pathways, have been observed in 1-year-old juvenile NHPs exposed to a maternal WSD, suggesting multiple systems are affected [11]. Recent data in humans also indicate that maternal obesity increases the capacity for umbilical cord mesenchymal stem cells to undergo adipogenesis, markers of which correlate with infant adiposity [12]. Consequently, there is an enormous public health need to target underlying dietary or metabolic factors in mothers with obesity or diabetes to prevent early-life obesity, NAFLD and diabetes risk in the next generation [3].

\section{Glossary}

Cross-fostering A technique in animal research in which offspring are removed from the biological mother at birth and breastfed by a surrogate mother

Dysbiosis An imbalance of or an unhealthy change in, the normal composition of microbes in a particular microbiome

Endotoxin Component of the outer membrane of Gramnegative bacteria known to elicit a strong immune response when in systemic circulation (endotoxaemia) also known as lipopolysaccharide

Enterohepatic circulation Circulation of substances such as bile acids, from the liver into bile, secretion into the small intestine, absorption by enterocytes and transportation back to the liver

Epigenome The chemical modification of the DNA (genome) (e.g. the attachment of a methyl group) to alter gene expression

Glycans Compounds consisting of a large number of monosaccharides linked glycosidically also known as polysaccharides glycans may also refer to the carbohydrate portion of a glycoconjugate (also known as glycoprotein glycolipid, proteoglycan or oligosaccharide)

Gnotobiotic mice Mice colonised by a known set of gut bacteria or no gut bacteria (germ-free) and raised in an aseptic environment

Inflammasome A multiprotein complex that activates caspases and leads to the formation and secretion of proinflammatory cytokines as a component of the innate immune system

Lamina propria Connective tissue layer of the intestine that together with the epithelium constitutes the gut mucosa

Lipopolysaccharide See Endotoxin

Microbiome The community of microorganisms found in a particular environment such as a part of the body

Oligosaccharide Saccharide polymer containing a small number of monosaccharides

Prebiotics Substances used to promote the growth of microorganisms often these are indigestible carbohydrates that are used as energy substrate for gut microbes

Probiotics Live bacteria and yeasts that are beneficial to a host

Short-chain fatty acid Saturated aliphatic organic acids with one to six carbons end-product of dietary fermentation by anaerobic microbiota

Toll-like receptor A component of the innate immune system that recognises structurally conserved motifs that are expressed exclusively on microbial pathogens to initiate an inflammatory response 


\section{Development of the infant microbiome and childhood obesity risk}

Evidence that the gut microbiota might influence obesity began with the observation that sterile, germ-free mice had decreased capacity for energy harvest compared with colonised counterparts [13]. Once colonised, these mice extracted more energy from dietary indigestible complex plant polysaccharides and increased their energy harvest, or caloric intake, contributing to obesity. More recently, human studies in twin pairs discordant for obesity demonstrated that transplanting microbiota from the twin with obesity, which demonstrated a shift towards increased Clostridia from the Firmicutes phylum as well as elevated Gammaproteobacteria and Deltaproteobacteria classes from the Proteobacteria phylum, into germ-free mice increased fat mass and biomarkers associated with the metabolic syndrome (refer to Fig. 1 for microbiota taxonomy) [14]. There is evidence that gut composition very early in life is correlated with later microbiota colonisation, suggesting that dysbiosis in infancy due to obesity exposure may have later consequences on metabolic health [15]. Groundbreaking studies in NHPs showed that a maternal high-fat diet (HFD) reduced the diversity of offspring intestinal microbiota in juvenile animals at 1 year of age [16], even after switching to a healthy diet at the time of weaning. This persistent effect of early-life diet suggests that maternal diet exposure during gestation and breastfeeding can pattern the composition of the microbial community, with long-lasting effects. However, studies assessing the duration of microbial disruption in these offspring is lacking. A review of the early infant microbiome and childhood obesity reported that despite a global reduction of the Bacteroidetes phylum, early elevations in the Bacteroides fragilis species and reductions in the genus Bifidobacterium correlated with elevated BMI in later childhood [15]. Further, the transition from a neonatal to a mature microbiota may be governed in part by the shift from a gut dominated by the Proteobacteria phylum, particularly of the Gammaproteobacteria class, to one dominated by the Bacteroidetes and Firmicutes phyla [17, 18]. In mouse [17] and human [18] studies, this pioneering Gammaproteobacteria, a driver of early inflammation necessary for adaptation of the innate immune system to prevent gastrointestinal autoimmunity, may be reduced in offspring exposed to maternal obesity. Cumulatively, these findings support the hypothesis that, although the early microbiome has erratic compositional shifts and high inter-individual differences before reaching a more stable microbiome at around 2 years of age $[19,20]$, there are clear implications for the early microbiome on life-long health.

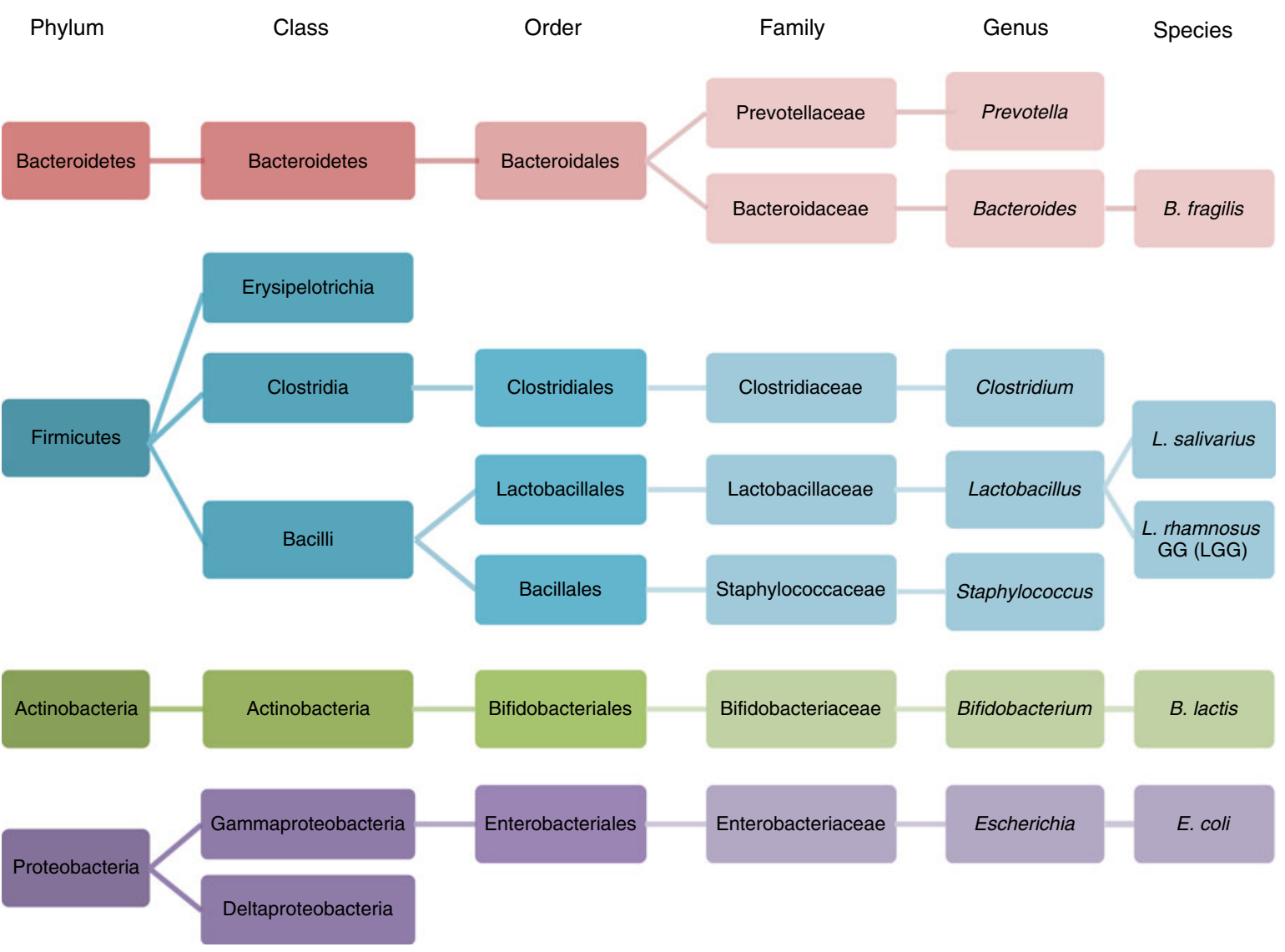

Fig. 1 Taxonomy of microorganisms referred to in this review article 


\section{Pregnancy and the maternal microbiome}

Each individual's gut microbiome consists of a unique pattern of microbes that is determined by both diversity (different strains of bacteria) and abundance (quantity of each strain). Mothers share their microbes and microbiome metabolites with the fetus in utero, during delivery and during lactation; the composition of the microbiome is shaped by the environment including diet, maternal health status and metabolism, mode of delivery, gestational weight gain (GWG), genetics and use of antibiotics.

The maternal gut microbiota differs between women with normal weight and obesity particularly in the latter half of pregnancy, with overweight women demonstrating increases in the Firmicutes phylum (Staphylococcus) as well as increases in some Proteobacteria (Escherichia coli) [21]. Furthermore, these differences are associated with increased neonatal birthweight $[21,22]$. One mechanism by which maternal microbes in obesity could affect the developing fetus is through elevated levels of microbe-derived plasma endotoxin; this may increase gut translocation of bacteria-derived products across the intestinal mucosa, which could contribute to systemic and placental inflammation and insulin resistance [23]. In 2012, Koren et al demonstrated changes in the maternal gut microbiome composition from the first to the third trimester of pregnancy [24]. When pooled stool samples from either trimester were inoculated into gnotobiotic mice, stool from the third trimester was associated with excess weight gain and inflammation compared with mice receiving stool from women in their first trimester. However, this pregnancy-related change in microbe composition has been challenged recently by DiGiulio et al [25] who, by repeated sampling throughout gestation, demonstrated that vaginal, gut and oral cavity microbiota remained relatively stable during and after pregnancy (except in those who experienced preterm delivery). These discrepant findings require independent confirmation and may be explained by heterogeneity in BMI, age, ethnicity, genetics, lifestyle, degree of maternal insulin resistance, gestational age and especially by differences in the maternal diet or supplementation, which could markedly affect microbiome composition [15].

\section{Mode of delivery affects the infant microbiome and obesity risk}

Although the founding microbiota composition in infants largely reflects maternal transfer during birth, the mode of delivery affects postnatal assembly of the microbiota and thereby altering resistance to pathogen invasion, immune stimulation and other important developmental cues early in life [26]. Infants born by vaginal delivery are colonised by microbes resident in the birth canal and the mother's gastrointestinal tract, whereas infants born by Caesarean delivery are initially colonised by skin flora $[26,27]$. In breastfed infants delivered at full term without medical interventions, the gut microbiota at 4 days is dominated by Gammaproteobacteria and some Staphylococcus species, followed by subsequent Bifidobacterium colonisation [18]. In babies born via Caesarean delivery, infants' stool revealed a decrease in Bacteroidetes phylum, Bifidobacterium and Lactobacillus species and a rise in Clostridium species, as well as an overall reduction in microbiome diversity; these differences persisted, in some cases even at 2 years of age $[26,28]$.

Differences in the early microbiome may have metabolic consequences, as Caesarean delivery is associated with a $46 \%$ increase in obesity risk at 7 years of age [29] and a $20 \%$ increased risk in development of type 1 diabetes compared with children born vaginally [30]. One study found that while most infants had acquired high levels of the genus Bifidobacterium by the age of 6 months, this acquisition was delayed in infants born via Caesarean delivery [27]. Furthermore, the abundance of these microbes inversely correlated with infant adiposity at the age of 18 months, demonstrating that the correlation of mode of delivery with infant adiposity may be driven by microbiome composition [27]. However, other confounding variables associated with Caesarean delivery risk, such as peripartum antibiotics, maternal obesity, glucose intolerance and insulin resistance, could independently influence the infant microbiome and obesity risk. Increased childhood BMI following Caesarean delivery is more pronounced in offspring of mothers with obesity [29], suggesting that gestational risk factors, mode of delivery and postnatal environment may contribute to shaping future obesity risk during this highly susceptible period of life.

While maternal vaginal and intestinal microbes are important, as they are the sites of vertical microbial transmission to the newborn during vaginal delivery, a recent paradigm shift suggests that the womb is not sterile and microbial exposure may begin in utero. The placenta and amniotic fluid have been reported to serve as potential sources of intrauterine microbial transmission from the mother to the developing fetus [31, 32]. However, the potential for contamination and the low biomass of microbes in these sources suggest that more research is needed to understand how they affect infant immunity and gut development.

\section{Maternal diet and infant breastfeeding shapes the microbial community: impact on immunity and metabolism}

In humans, the composition of breast milk influences the development of intestinal permeability, energy retention 
capacity and autoimmunity and may differ according to maternal obesity status and maternal diet [33]. Accumulating evidence suggests that exclusive breastfeeding is associated with lower risk of paediatric obesity [34, 35] and that this protection may be even greater in women with obesity who breastfeed their infants for at least 6 months [36]. However, breastfeeding-induced protection against obesity in offspring is not universal across all lactating mothers and remains poorly characterised [36].

Cross-fostering studies in mice in which offspring of lean dams were nursed by obese dams suggested that maternal obesity had distinct effects on the hormonal, nutritional and microbial composition of milk that were associated with a NAFLD phenotype as well as with increased body weight, plasma insulin and proinflammatory cytokines in offspring [37]. However, it is important to note that in mouse studies, the effect of coprophagy (the eating of faeces) cannot be ruled out as a contributing factor in offspring response to obese dam exposure. Similarly, offspring born to mothers fed a WSD and switched to normal chow diet after weaning had worse outcomes than those offspring whose mothers were only exposed to standard low-fat diet with respect to infection, autoimmunity and allergic sensitisation, which often precede the development of obesity [38]. These maternal WSD effects were linked to alterations of the offspring gut microbiome, suggesting that excess dietary fat consumption during pregnancy and lactation can have effects on offspring immunity and gut barrier function that persist into later life. However, very few studies take into consideration differences in breast milk related to maternal phenotype and even fewer link these to their impact on infant microbiome and infant gut function. The factors involved in human milk transfer of obesity risk remain poorly understood.

In humans, breast milk serves as a principal source of important immune and growth factors necessary for initial microbial colonisation. Human milk glycans are a large and diverse group of polysaccharides that include free human milk oligosaccharides (HMOs), glycoproteins, glycopeptides and glycolipids capable of enriching populations of Bifidobacteria [39]. HMOs are complex sugars that resist digestion by the infant's stomach and are metabolised by selective intestinal microbiota. The metabolism of HMOs can lead to the production of short-chain fatty acids (SCFAs), which may have metabolic influences (discussed below). It is generally accepted that HMOs have significant prebiotic effects, serving as a source of energy and nutrients that enable desired bacteria to colonise the infant intestinal tract [40]. The type of oligosaccharides that infants receive from their mothers depends on the mother's genotype and phenotype $[33,41]$ but the amount may be variable, particularly given that mothers with obesity and diabetes have difficulties breastfeeding. A more in-depth coverage of milk glycans and the infant gut microbiome is beyond the scope of this review but this topic is covered extensively by Bode [40].

The most common microbes found in human breast milk, but in relatively low abundance, are from the genera Bifidobacterium and Lactobacillus [42], similar to the microbes found in the infant gut. This suggests that breast milk microbiota, albeit at very low concentrations, can be transferred to the infant gut, although this finding is not universal [43]. Similar to the gut microbiome, breast milk microbial communities from mothers with obesity or excessive GWG were less diverse and had a higher relative abundance of the groups Lactobacillus and Staphylococcus and reduced Bifidobacterium at 6 months post delivery [44]. Moreover, breast milk from women with obesity contains high levels of metabolic and inflammatory markers, such as leptin, insulin, glucose, IL-6 and TNF $\alpha$, compared with milk from women with normal weight [45]. These changes, in addition to changes in antioxidant and microbial status, may be important factors in shaping the early infant gut microbiome [46].

Medium-chain saturated and long-chain unsaturated fatty acids present in breast milk have also been shown to have antimicrobial properties [47], suggesting that higher triacylglycerol hydrolysis or circulating fatty acids in obesity might have an impact on microbial colonisation in the infant. Studies are needed to establish whether human milk lipids have an antimicrobial effect in the offspring and whether they play a role in the development of the infant microbiome. To date very little is known about the contents of breast milk from women with diabetes [48, 49]. Compared with breastfed infants, the diversity of the genus Bifidobacterium was found to be decreased in formula-fed infants [50] and this was associated with increased adiposity at 18 months [27]. Interestingly, formula-fed infants have greater diversity of gut microbial communities relative to breastfed infants and, functionally, these infants have higher proportions of proinflammatory Gammaproteobacteria [20] which may suggest delayed gut maturation. It is important to note that thus far studies have not addressed the effect of breast milk volume consumed, duration of breastfeeding or the impact of formula supplementation on the development of the infant gut microbiome. Given the beneficial attributes of breast milk in establishing the early microbiome, a deviation from the normal composition of breast milk may be associated with an increased risk of obesity and inflammatory conditions. Thus, while breastfeeding is strongly recommended as the best source of nutrition for a newborn, it is clear that not all breast milk is the same. This raises the question of whether and how changes in diet or pre- or probiotics in lactating women with obesity or diabetes can affect the developing gut microbiome of the newborn (discussed below). 


\section{Early antibiotic exposure increases the risk for obesity}

Retrospective studies of antibiotic exposure at various windows of time during the first 2 years of life found that broad-spectrum treatment is associated with childhood obesity $[51,52]$. Children born to mothers who were given antibiotics during the second or third trimester of pregnancy had an $84 \%$ higher risk of obesity at age 7 years; however, first-trimester antibiotics had no effect [29]. Animal studies have suggested that there is a critical window during which alteration of the gut microbiota can influence future health. Antibiotic treatment given to neonatal mice just prior to or immediately following birth through weaning resulted in enhanced susceptibility to allergic asthma, while the same treatment in adult mice had no effect [53-55]. Likewise, mice exposed to prenatal antibiotics had increased body fat percentage and increased expression of adipogenic targets, including peroxisome proliferator-activated receptor $\gamma$ and fatty acid binding protein 2. Moreover, in mice, a HFD combined with maternal antibiotic use exacerbated changes in body mass and hyperinsulinaemia in the offspring, suggesting that maternal obesity may compound the effect of antibiotics on disruption of the microbiome and downstream metabolic outcomes [54]. Studies of infant postnatal antibiotic exposure in the first 6-12 months of life have found an association between antibiotic use and elevated weight and central adiposity as early as 24 months and up to 9 years of age regardless of maternal weight status $[52,56,57]$. These studies point to the ability of both maternal microbiome and early infant antibiotic exposure to program metabolic pathways in infants. The early gut microbiome and the immune system is reviewed more comprehensively by Maynard et al [58].

\section{The microbiome and GDM}

GDM is a growing problem and has a current prevalence of $9.2 \%$ in the USA [59] and 2-6\% in Europe [60]. The characterisation and role of microbiomes of women with GDM and their offspring are understudied. Data characterising intestinal microbiota in patients with type 2 diabetes showed that in addition to reduction of the Firmicutes phylum, the ratio of Bacteroidetes to Firmicutes correlated positively and significantly with plasma glucose concentration but not with BMI [61]. Thus, bacterial species, specific for type 2 diabetes rather than obesity, can be considered as a separate signature altered by hyperglycaemia. More recently, the stool microbiota of insulin-resistant women with a history of GDM was characterised at 3-16 months postpartum and compared with that of normoglycaemic postpartum controls [62]. These authors found that a subset of individuals with former GDM had relatively higher abundance of the Prevotellaceae family despite no apparent differences in diet compared with the control group. Additionally, at the phylum level, the formerGDM group had reduced abundance of Firmicutes, similar to the situation in type 2 diabetes. A role for Prevotella in mucin degradation in the gut [63] suggests that changes in gut health could be present in individuals who have high levels of insulin resistance, but this hypothesis has not been tested directly in individuals matched for similar BMI, race or breastfeeding status. In one study, offspring of women with diabetes, the meconium (the first intestinal discharge of a newborn) demonstrated an increased within-sample diversity and Bacteroidetes relative abundance compared with meconium from infants born to mothers without diabetes [31]. However, the shift in meconium microbes was most apparent in those with pre-pregnancy diabetes, not GDM. The authors of this study hypothesised that certain conditions, such as pre-existing diabetes, may make microbes more transmissible in utero [31]; however, cross-fostering studies in rats have pointed to a specific role of breast milk in the transfer of GDM morbidity to offspring, particularly on neurological development [64]. Although correlations between GDM and infant outcomes exist, the gut microbial shifts in GDM remain to be characterised before mechanisms of cause and effect on infant health can be elucidated.

\section{Mechanisms for microbiome-induced metabolic disorders}

SCFAs SCFAs have recently emerged as pivotal regulators of host metabolism and immunity. SCFAs are gut microbiome byproducts, mainly acetates, propionates and butyrates, produced through anaerobic fermentation of indigestible dietary-fibre carbohydrates $[65,66]$. SCFAs are absorbed and used by colonocytes (butyrate) and peripheral tissues (acetate) for energy, or may act as substrates for lipogenesis (acetate), gluconeogenesis (propionate) or regulation of cholesterol synthesis (propionate) in the liver. The hypothesis that dysbiosis may result in increased energy harvest was tested in subjects with normal weight and obesity that were fed the same diet for 3 days which led to increased energy retention of $\sim 630 \mathrm{~kJ}(\sim 150 \mathrm{kcal})$ in individuals with obesity and increased abundance of members of the Firmicutes phylum and reduced abundance of Bacteroidetes in stool samples in response to various diets [67]. This shift was also associated with elevated SCFAs. Faecal metabolites in overweight children support this hypothesis since overweight as opposed to normal-weight children have lower levels of intermediate metabolites such as lactate, yet higher levels of butyrate, which is the byproduct of lactate-utilising microbiota, suggesting extensive substrate utilisation. In addition to increased energy extraction from the diet, SCFA metabolites can also influence levels of satiety hormone which could lead 
to altered food intake. Another mechanism by which SCFAs alter host physiology is through modification of immune cells in the gut. SCFAs upregulate colonic T regulatory (Treg) cells and render lamina propria macrophages hyporesponsive to normal gut microbes through the downregulation of proinflammatory effectors, shifting the cells toward an anti-inflammatory phenotype $[66,68]$.

Although levels of SCFAs have been reported to be elevated in faecal samples from individuals with obesity [69], it remains to be seen whether this is a cause of or response to the metabolic and inflammatory shifts in the microbiome. Knockdown of the SCFA receptors, namely G proteincoupled receptors (GPR40, GPR41 and GPR43), has led to inflammation, glucose intolerance and diet-induced obesity in mouse models [70]. Moreover, studies of SCFAs have demonstrated beneficial metabolic effects when delivered enterally [71]. In pregnant mice, supplementation with butyrate reduced maternal proinflammatory factors TNF $\alpha$ and IL- $1 \beta$ as well as weight gain, plasma glucose, insulin, triacylglycerol and cholesterol [72]; these effects may have been be due to butyrate inhibiting the inflammation and lipolysis generated by the interaction of adipocytes and macrophages [73]. Furthermore, dietary butyrate ameliorated HFD-induced pancreatic beta cell dysfunction, as manifested by increased insulin storage, beta cell size, mass and apoptosis [72]. SCFAs also act as important microbial signals to remodel the intestinal microbial community through activation of the inflammasome [74], thereby helping prevent chronic intestinal inflammatory responses to microbes and their products. Finally, the discovery of uteroplacental GPR41 and GPR43 receptors and their role in the inflammatory processes of labour could provide a path by which SCFAs influence fetal programming through their action on the placenta [75].

Epigenetics Recent studies have shown that microbes affect a diverse set of epigenetic factors, including DNA methylation, histone modification, chromatin-associated complexes and non-coding RNAs, to alter chromatin structure and gene expression [76]. SCFAs have been reported to influence the epigenome; specifically, butyrate is recognised as a potent histone deacetylase inhibitor $[66,77]$. Butyrate exerts an anti-inflammatory effect in mice by promoting Treg cell differentiation through an increase in histone $\mathrm{H} 3$ acetylation, which corresponds to increased Foxp3 mRNA expression $[78,79]$. The microbiota in mice is also required to modulate the expression of Toll-like receptors 2 and 4 in the colon through epigenetic mechanisms [80]. Interestingly, a recent pilot study correlated pregnant women with either Firmicutes or Bacteroidetes as a dominant group with differential DNA methylation profiles of gene promoters involved in lipid metabolism, obesity and inflammation in whole blood collected from the same women at 6 months postpartum [81], suggesting a relationship between microbes, or their products, and host epigenetic regulation.

Bile acids Another important function of the gut microbiome that may affect infant physiology is the metabolism of primary bile acids (BAs) to secondary BAs in the small intestine. While $\sim 95 \%$ of secreted BAs is reabsorbed from the small intestine via the enterohepatic circulation pathway, $\sim 5 \%$ reaches the colon, where they are excreted. The primary BAs cholic acid and chenodeoxycholic acid are synthesised from cholesterol in the liver and conjugated to taurine or glycine to form bile salts before secretion into the duodenum where the bile assists in lipid digestion [82]. Bacterial salt hydrolase deconjugates and dehydroxylates primary BAs to form secondary BAs, most commonly deoxycholic acid and lithocholic acid [82]. Secondary BAs activate nuclear farnesoid $X$ receptors (FXRs) in the liver, which regulate liver BA synthesis [83] and $G$ protein-coupled BA receptor 1 (GPBAR1/TGR5) in the gut; this enhances secretion of glucagon-like peptide-1, which potentiates glucose-induced insulin secretion from beta cells [84]. The beneficial effects of BA signalling via FXR and TGR5 also include attenuation of proinflammatory innate immune responses in a mouse model of NAFLD [85], lipopolysaccharide (LPS)-induced hepatic damage and inflammation [86] and inflammatory bowel disease [87]. Conversely, there is evidence that excess BAs may influence microbiota composition through their strong antimicrobial activity. In mice, overfeeding with BAs induced changes similar to those seen with a HFD, including a shift from Bacteroidetes to Firmicutes (specifically the Clostridia and Erysipelotrichia classes) [88]. The role of microbiome development and BA production in early life on glucose homeostasis and inflammation warrants further investigation.

\section{Prevention and intervention with pre- and probiotics in mothers and infants}

The use of pre- and probiotics has been shown to rapidly modify the microbial community and reduce (at least temporarily) adiposity and chronic inflammation in animal models of obesity and in limited human studies [89-91]. There have only been a handful of randomised controlled trials (RCTs) that examined the effects of probiotics administered during pregnancy with the aim of improving insulin sensitivity and reducing GDM diagnosis, with limited success. In one RCT either Bifidobacterium lactis alone or B. lactis plus Lactobacillus rhamnosus GG (LGG) probiotic was administered to pregnant women 14 days before a scheduled Caesarean delivery [92]. Both treatments (compared with placebo) were found to decrease the expression of Toll-like receptor genes in the placenta and meconium of neonates, 
suggesting that in utero microbial exposure can alter the fetal innate immune system development. A Finnish study randomised 256 women during their first trimester of pregnancy into a control or dietary intervention group [93]. The intervention group received intensive dietary counselling (increased fibre, less fat) provided by a nutritionist and were further randomised, double-blind, to receive a daily LGG and $B$. lactis probiotic that continued until the end of exclusive breastfeeding (average 3.4 months) postpartum. The infants were followed up at 24 months of age. Dietary counselling combined with probiotics decreased the frequency of GDM from $36 \%$ in the diet/placebo group to $13 \%$. Despite lowering the risk of GDM, there were no significant differences in birthweight among groups after controlling for maternal BMI, smoking, length of gestation or infant growth over 24 months. This study lacked confirmation of changes in maternal or infant microbes following probiotic consumption. Interestingly, the risk of central adiposity in mothers at 6 months postpartum, defined as waist circumference $80 \mathrm{~cm}$ or more, was lower in women in the diet/probiotics group compared with the control/placebo group, with no effect seen after dietary intervention alone [94].
In a more recent RCT 175 pregnant women with an early pregnancy BMI of 30.0-39.9 kg/m $\mathrm{m}^{2}$ were assigned to receive either a daily Lactobacillus salivarius probiotic or a placebo capsule from 24 to 28 weeks of gestation in addition to routine antenatal care [95]. From pre-intervention to post-intervention, comparing groups there were no changes in fasting glucose, incidence of GDM or neonatal anthropometric measures [95]. However, it is possible that the differences between studies may be due to differences in timing, duration and type of probiotic supplementation used, as well as a lack of analysis of the potential dietary influence on probiotic effects, rather than a lack of probiotic capacity to effect maternal obesity and GDM.

Although studies of maternal probiotic supplementation have not found differences in infant anthropomorphic outcomes $[93,95,96]$, some studies have reported differences in infant gut microbial composition [96-98]. An RCT involving 122 mother-infant pairs at high risk of developing allergic disease found that daily LGG and B. lactis supplementation from 36 weeks gestation to delivery resulted in higher Bifidobacterium colonisation in the infant at 90 days of life despite the lack of LGG in maternal milk samples 1 month
Fig. 2 Proposed pathways for the transgenerational cycle of obesity. GWG, pre-pregnancy BMI, development of GDM and/or HFD/WSD can result in maternal gut dysbiosis. This dysbiosis may be directly transmitted to the infant and may cause dysbiosis in the infant gut by causing alterations to SCFA metabolite production, a proinflammatory state, epigenetic alterations and increased energy extraction from ingested nutrients. External influences such as early-life nutrition (breastfeeding vs formula-feeding), mode of delivery and antibiotic treatment may additionally influence the composition of the infant gut microbiome. These changes in gut microbiome function may result in infants born large for gestational age and with excess adiposity, both of which place the child at increased risk of obesity, immune dysfunction and NAFLD later in life. Adulthood obesity during childbearing years then perpetuates the cycle of obesity. DM, type 1 or 2 diabetes

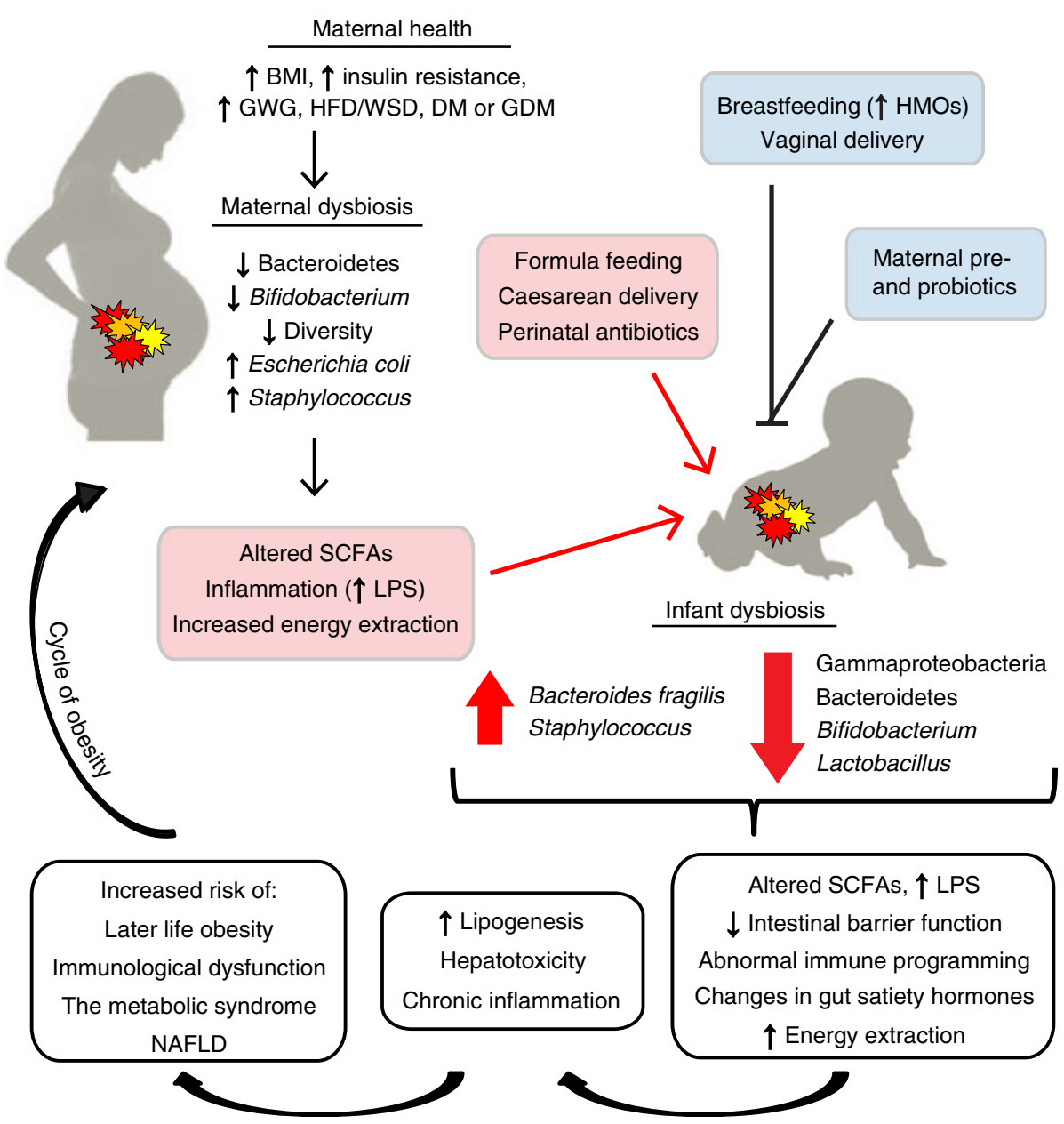


postpartum [96]. A retrospective study of six women taking LGG from 30-36 weeks gestation until delivery found that in vaginally delivered infants, colonisation of LGG persisted up to 6 months postpartum [97]. Interestingly, a subset of infants remained colonised up to 24 months despite a lack of maternal LGG supplementation at 1 month postpartum. However, anthropometric and metabolic outcomes were not assessed in the infants. Of note, prenatal LGG administration, while modulating Bifidobacterium levels, did not result in an increase in overall infant microbiome diversity at 7 days of life [99]. While these studies cumulatively support the ability of maternal probiotic supplementation to alter the infant gut microbiome independent of direct vertical transmission of microbes, the effect of supplement timing during gestation, characterisation of these alterations, what specific factor(s) influence the duration of effect, concurrent infant diet influences and their long-term outcomes deserve further examination.

\section{Perspectives}

Pregnancy and the postnatal period are critical windows of opportunity for the prevention of metabolic diseases in the next generation. Maternal obesity or diabetes, along with poor diet, may affect the establishment of a stable, healthy intestinal microbiome in infants, with long-term health consequences. Early communication between the infant host and its gut microbiota can affect the programming of immune cells and other metabolic and biochemical pathways that may greatly affect the developmental trajectory of body weight. Bacteriadriven inflammation can reduce the abundance of other bacteria that compete with the proinflammatory pathogens, promoting organisms that may increase the ability of the gut microbiota to extract otherwise indigestible dietary polysaccharides. Alternatively, enteroendocrine cells of the gut modify or secrete a variety of bioactive peptides and fatty acids, all known to be connected to food intake, lipid storage and energy homeostasis, which can be influenced by microbial metabolites. At present, there are no well-controlled longitudinal studies of the microbiome in infants of women with diabetes during pregnancy (a growing population). Microbiota specific for maternal diabetes rather than obesity may emerge in the infant as a separate signature altered by hyperglycaemia that elicits additional risk factors for infant health.

The founding microbial composition in infants born to mothers with obesity or diabetes, and the bioactive components of breast milk, are just beginning to be explored for their relationships to infant body composition. However, gut microbiota composition and metabolic biomarkers for obesity in adulthood may not be applicable to the rapidly developing infant microbiome, which may need to respond differentially in order to develop immune tolerance and respond to prenatal exposures, birth events and powerful postnatal dietary and environmental factors. Prospective follow-up studies that can account for multiple influential covariates, including ethnicity, sex of offspring, geographical location, metabolic and immune health of the mother and infant, and diet using more standardised profiling techniques are critical for understanding whether early microbiome development has a causal role in obesity risk. A skewed early-life gut microbiota composition could likely play a pivotal role in the mechanism(s) underlying the additional obesity risk in infants of mothers with obesity or diabetes, as illustrated in Fig. 2. Despite important evidence showing associated changes in maternal and infant nutrition with obesity and dynamic changes in the infant microbiome, the causative mechanisms in the newborn infant are still unknown. Consequently, understanding the mechanisms whereby changes in maternal and infant diet alter the composition and output of the early microbiome may allow us a potentially powerful opportunity for primary prevention in mothers and infants to reduce long-term metabolic risks in the next generation.

Acknowledgements We thank R. C. Janssen (Department of Pediatrics, University of Colorado Anschutz Medical Campus) for help with editing the manuscript and all members of the Friedman laboratory for insightful discussions.

Funding This work was supported by the National Institutes of Health, the American Diabetes Association and the Colorado Nutrition and Obesity Research Center (NORC).

Duality of interest The authors declare that there is no duality of interest associated with this manuscript.

Contribution statement All authors researched, wrote, contributed to discussion and reviewed and/or edited the manuscript. All authors approved the final version of the manuscript.

\section{References}

1. Heerwagen MJ, Miller MR, Barbour LA, Friedman JE (2010) Maternal obesity and fetal metabolic programming: a fertile epigenetic soil. Am J Physiol Regul Integr Comp Physiol 299:R711R722

2. Nolan CJ (2013) Normal long-term health for infants of diabetic mothers: can we achieve it? J Clin Endocrinol Metab 98:35923594

3. Friedman JE (2015) Obesity and gestational diabetes mellitus pathways for programming in mouse, monkey, and man - where do we go next? The 2014 Norbert Freinkel Award Lecture. Diabetes Care 38:1402-1411

4. Barbour LA (2014) Changing perspectives in pre-existing diabetes and obesity in pregnancy: maternal and infant short- and long-term outcomes. Curr Opin Endocrinol Diabetes Obes 21:257-263 
5. Weng SF, Redsell SA, Nathan D, Swift JA, Yang M, Glazebrook C (2013) Estimating overweight risk in childhood from predictors during infancy. Pediatrics 132:e414-e421

6. Boney CM, Verma A, Tucker R, Vohr BR (2005) Metabolic syndrome in childhood: association with birth weight, maternal obesity, and gestational diabetes mellitus. Pediatrics 115:e290-e296

7. McCurdy CE, Bishop JM, Williams SM et al (2009) Maternal highfat diet triggers lipotoxicity in the fetal livers of nonhuman primates. J Clin Invest 119:323-335

8. Thorn SR, Baquero KC, Newsom SA et al (2014) Early life exposure to maternal insulin resistance has persistent effects on hepatic NAFLD in juvenile nonhuman primates. Diabetes 63:2702-2713

9. Brumbaugh DE, Tearse P, Cree-Green M et al (2013) Intrahepatic fat is increased in the neonatal offspring of obese women with gestational diabetes. J Pediatr 162:930-936

10. Brumbaugh DE, Friedman JE (2014) Developmental origins of nonalcoholic fatty liver disease. Pediatr Res 75:140-147

11. Sullivan EL, Nousen EK, Chamlou KA, Grove KL (2012) The impact of maternal high-fat diet consumption on neural development and behavior of offspring. Int J Obes Suppl 2:S7-S13

12. Boyle KE, Patinkin ZW, Shapiro ALB, Baker PR II, Dabelea D, Friedman JE (2015) Mesenchymal stem cells from infants born to obese mothers exhibit greater potential for adipogenesis: the Healthy Start BabyBUMP Project. Diabetes. doi:10.2337/db150849

13. Turnbaugh PJ, Ley RE, Mahowald MA, Magrini V, Mardis ER, Gordon JI (2006) An obesity-associated gut microbiome with increased capacity for energy harvest. Nature 444:1027-1031

14. Ridaura VK, Faith JJ, Rey FE et al (2013) Gut microbiota from twins discordant for obesity modulate metabolism in mice. Science 341:1241214

15. Koleva PT, Bridgman SL, Kozyrskyj AL (2015) The infant gut microbiome: evidence for obesity risk and dietary intervention. Nutrients 7:2237-2260

16. Ma J, Prince AL, Bader D et al (2014) High-fat maternal diet during pregnancy persistently alters the offspring microbiome in a primate model. Nat Commun 5:3889

17. Mirpuri J, Raetz M, Sturge CR et al (2014) Proteobacteria-specific IgA regulates maturation of the intestinal microbiota. Gut Microbes 5:28-39

18. Eggesbø M, Moen B, Peddada S et al (2011) Development of gut microbiota in infants not exposed to medical interventions. APMIS 119:17-35

19. Koenig JE, Spor A, Scalfone N et al (2011) Succession of microbial consortia in the developing infant gut microbiome. Proc Natl Acad Sci U S A 108:4578-4585

20. Bäckhed F, Roswall J, Peng Y et al (2015) Dynamics and stabilization of the human gut microbiome during the first year of life. Cell Host Microbe 17:690-703

21. Santacruz A, Collado MC, García-Valdés L et al (2010) Gut microbiota composition is associated with body weight, weight gain and biochemical parameters in pregnant women. Br J Nutr 104:83-92

22. Collado MC, Isolauri E, Laitinen K, Salminen S (2008) Distinct composition of gut microbiota during pregnancy in overweight and normal-weight women. Am J Clin Nutr 88:894-899

23. Basu S, Haghiac M, Surace P et al (2011) Pregravid obesity associates with increased maternal endotoxemia and metabolic inflammation. Obesity 19:476-482

24. Koren O, Goodrich JK, Cullender TC et al (2012) Host remodeling of the gut microbiome and metabolic changes during pregnancy. Cell 150:470-480

25. DiGiulio DB, Callahan BJ, McMurdie PJ et al (2015) Temporal and spatial variation of the human microbiota during pregnancy. Proc Natl Acad Sci U S A 112:11060-11065

26. Dominguez-Bello MG, Costello EK, Contreras M et al (2010) Delivery mode shapes the acquisition and structure of the initial microbiota across multiple body habitats in newborns. Proc Natl Acad Sci U S A 107:11971-11975

27. Dogra S, Sakwinska O, Soh SE et al (2015) Dynamics of infant gut microbiota are influenced by delivery mode and gestational duration and are associated with subsequent adiposity. MBio 6, e02419

28. Azad MB, Konya T, Maughan H et al (2013) Gut microbiota of healthy Canadian infants: profiles by mode of delivery and infant diet at 4 months. CMAJ 185:385-394

29. Mueller NT, Whyatt R, Hoepner L et al (2015) Prenatal exposure to antibiotics, cesarean section and risk of childhood obesity. Int $\mathrm{J}$ Obes (Lond) 39:665-670

30. Cardwell CR, Stene LC, Joner G et al (2008) Caesarean section is associated with an increased risk of childhood-onset type 1 diabetes mellitus: a meta-analysis of observational studies. Diabetologia 51: 726-735

31. Hu J, Nomura Y, Bashir A et al (2013) Diversified microbiota of meconium is affected by maternal diabetes status. PLoS One 8 , e78257

32. Aagaard K, Ma J, Antony KM, Ganu R, Petrosino J, Versalovic J (2014) The placenta harbors a unique microbiome. Sci Transl Med 6:237ra265

33. Savino F, Benetti S, Liguori SA, Sorrenti M, Cordero Di Montezemolo L (2013) Advances on human milk hormones and protection against obesity. Cell Mol Biol 59:89-98

34. Yan J, Liu L, Zhu Y, Huang G, Wang PP (2014) The association between breastfeeding and childhood obesity: a meta-analysis. BMC Public Health 14:1267

35. Young BE, Johnson SL, Krebs NF (2012) Biological determinants linking infant weight gain and child obesity: current knowledge and future directions. Adv Nutr 3:675-686

36. Woo JG, Martin LJ (2015) Does breastfeeding protect against childhood obesity? Moving beyond observational evidence. Curr Obes Rep 4:207-216

37. Oben JA, Mouralidarane A, Samuelsson AM et al (2010) Maternal obesity during pregnancy and lactation programs the development of offspring non-alcoholic fatty liver disease in mice. J Hepatol 52: 913-920

38. Myles IA, Fontecilla NM, Janelsins BM, Vithayathil PJ, Segre JA, Datta SK (2013) Parental dietary fat intake alters offspring microbiome and immunity. J Immunol 191:3200-3209

39. Zivkovic AM, German JB, Lebrilla CB, Mills DA (2011) Human milk glycobiome and its impact on the infant gastrointestinal microbiota. Proc Natl Acad Sci U S A 108:4653-4658

40. Bode L (2009) Human milk oligosaccharides: prebiotics and beyond. Nutr Rev 67:S183-S191

41. Wacklin P, Mäkivuokko H, Alakulppi N et al (2011) Secretor genotype (FUT2 gene) is strongly associated with the composition of Bifidobacteria in the human intestine. PLoS One 6, e20113

42. Martin R, Jimenez E, Heilig H et al (2009) Isolation of bifidobacteria from breast milk and assessment of the bifidobacterial population by PCR-denaturing gradient gel electrophoresis and quantitative real-time PCR. Appl Environ Microbiol 75:965-969

43. Hunt KM, Foster JA, Forney LJ et al (2011) Characterization of the diversity and temporal stability of bacterial communities in human milk. PLoS One 6, e21313

44. Cabrera-Rubio R, Collado MC, Laitinen K, Salminen S, Isolauri E, Mira A (2012) The human milk microbiome changes over lactation and is shaped by maternal weight and mode of delivery. Am J Clin Nutr 96:544-551

45. Young BE, Morrow A, Davidson B, Geraghty S, Patinkin ZW, Krebs NF (2014) 4-Hydroxynonenol is present in human milk and related to gestational age at delivery and excessive infant weight gain. FASEB J 28:247.246 
46. Fields DA, Demerath EW (2012) Relationship of insulin, glucose, leptin, IL-6 and TNF-alpha in human breast milk with infant growth and body composition. Pediatr Obes 7:304-312

47. Thormar H, Isaacs CE, Brown HR, Barshatzky MR, Pessolano T (1987) Inactivation of enveloped viruses and killing of cells by fatty acids and monoglycerides. Antimicrob Agents Chemother 31:2731

48. Matias SL, Dewey KG, Quesenberry CP Jr, Gunderson EP (2014) Maternal prepregnancy obesity and insulin treatment during pregnancy are independently associated with delayed lactogenesis in women with recent gestational diabetes mellitus. Am J Clin Nutr 99:115-121

49. Stuebe AM (2015) Does breastfeeding prevent the metabolic syndrome, or does the metabolic syndrome prevent breastfeeding? Semin Perinatol 39:290-295

50. Roger LC, Costabile A, Holland DT, Hoyles L, McCartney AL (2010) Examination of faecal Bifidobacterium populations in breast- and formula-fed infants during the first 18 months of life. Microbiology 156:3329-3341

51. Bailey LC, Forrest CB, Zhang P, Richards TM, Livshits A, DeRusso PA (2014) Association of antibiotics in infancy with early childhood obesity. JAMA Pediatr 168:1063-1069

52. Azad MB, Bridgman SL, Becker AB, Kozyrskyj AL (2014) Infant antibiotic exposure and the development of childhood overweight and central adiposity. Int J Obes (Lond) 38:1290-1298

53. Cho I, Yamanishi S, Cox L et al (2012) Antibiotics in early life alter the murine colonic microbiome and adiposity. Nature 488:621-626

54. Cox LM, Yamanishi S, Sohn J et al (2014) Altering the intestinal microbiota during a critical developmental window has lasting metabolic consequences. Cell 158:705-721

55. Nobel YR, Cox LM, Kirigin FF et al (2015) Metabolic and metagenomic outcomes from early-life pulsed antibiotic treatment. Nat Commun 6:7486

56. Ajslev TA, Andersen CS, Gamborg M, Sorensen TI, Jess T (2011) Childhood overweight after establishment of the gut microbiota: the role of delivery mode, pre-pregnancy weight and early administration of antibiotics. Int J Obes (Lond) 35:522-529

57. Trasande L, Blustein J, Liu M, Corwin E, Cox LM, Blaser MJ (2013) Infant antibiotic exposures and early-life body mass. Int J Obes (Lond) 37:16-23

58. Maynard CL, Elson CO, Hatton RD, Weaver CT (2012) Reciprocal interactions of the intestinal microbiota and immune system. Nature 489:231-241

59. DeSisto CL, Kim SY, Sharma AJ (2014) Prevalence estimates of gestational diabetes mellitus in the United States, Pregnancy Risk Assessment Monitoring System (PRAMS), 2007-2010. Prev Chronic Dis 11, E104

60. Buckley BS, Harreiter J, Damm P et al (2012) Gestational diabetes mellitus in Europe: prevalence, current screening practice and barriers to screening. A review. Diabet Med 29:844-854

61. Larsen N, Vogensen FK, van den Berg FW et al (2010) Gut microbiota in human adults with type 2 diabetes differs from non-diabetic adults. PLoS One 5, e9085

62. Fugmann M, Breier M, Rottenkolber $M$ et al (2015) The stool microbiota of insulin resistant women with recent gestational diabetes, a high risk group for type 2 diabetes. Sci Rep 5:13212

63. Wright DP, Rosendale DI, Robertson AM (2000) Prevotella enzymes involved in mucin oligosaccharide degradation and evidence for a small operon of genes expressed during growth on mucin. FEMS Microbiol Lett 190:73-79

64. Fahrenkrog S, Harder T, Stolaczyk E et al (2004) Cross-fostering to diabetic rat dams affects early development of mediobasal hypothalamic nuclei regulating food intake, body weight, and metabolism. J Nutr 134:648-654
65. Priyadarshini M, Thomas A, Reisetter AC et al (2014) Maternal short-chain fatty acids are associated with metabolic parameters in mothers and newborns. Transl Res 164:153-157

66. Park J, Kim M, Kang SG et al (2015) Short-chain fatty acids induce both effector and regulatory $\mathrm{T}$ cells by suppression of histone deacetylases and regulation of the mTOR-S6K pathway. Mucosal Immunol 8:80-93

67. Jumpertz R, Le DS, Turnbaugh PJ et al (2011) Energy-balance studies reveal associations between gut microbes, caloric load, and nutrient absorption in humans. Am J Clin Nutr 94:58-65

68. Chang PV, Hao L, Offermanns S, Medzhitov R (2014) The microbial metabolite butyrate regulates intestinal macrophage function via histone deacetylase inhibition. Proc Natl Acad Sci U S A 111: 2247-2252

69. Schwiertz A, Taras D, Schäfer K et al (2010) Microbiota and SCFA in lean and overweight healthy subjects. Obesity (Silver Spring) 18: 190-195

70. Bellahcene M, O'Dowd JF, Wargent ET et al (2013) Male mice that lack the G-protein-coupled receptor GPR41 have low energy expenditure and increased body fat content. Br J Nutr 109:1755-1764

71. den Besten G, Bleeker A, Gerding A et al (2015) Short-chain fatty acids protect against high-fat diet-induced obesity via a PPAR $\gamma$ dependent switch from lipogenesis to fat oxidation. Diabetes 64: 2398-2408

72. Li HP, Chen X, Li MQ (2013) Butyrate alleviates metabolic impairments and protects pancreatic beta cell function in pregnant mice with obesity. Int J Clin Exp Pathol 6:1574-1584

73. Ohira H, Fujioka Y, Katagiri C et al (2013) Butyrate attenuates inflammation and lipolysis generated by the interaction of adipocytes and macrophages. J Atheroscler Thromb 20:425-442

74. Macia L, Tan J, Vieira AT et al (2015) Metabolite-sensing receptors GPR43 and GPR109A facilitate dietary fibre-induced gut homeostasis through regulation of the inflammasome. Nat Commun 6: 6734

75. Voltolini C, Battersby S, Etherington SL, Petraglia F, Norman JE, Jabbour HN (2012) A novel antiinflammatory role for the shortchain fatty acids in human labor. Endocrinology 153:395-403

76. Takahashi K (2014) Influence of bacteria on epigenetic gene control. Cell Mol Life Sci 71:1045-1054

77. Sealy L, Chalkley R (1978) The effect of sodium butyrate on histone modification. Cell 14:115-121

78. Furusawa Y, Obata Y, Fukuda S et al (2013) Commensal microbederived butyrate induces the differentiation of colonic regulatory $\mathrm{T}$ cells. Nature 504:446-450

79. Arpaia N, Campbell C, Fan X et al (2013) Metabolites produced by commensal bacteria promote peripheral regulatory $\mathrm{T}$ cell generation. Nature 504:451-455

80. Kellermayer R, Dowd SE, Harris RA et al (2011) Colonic mucosal DNA methylation, immune response, and microbiome patterns in Toll-like receptor 2-knockout mice. FASEB J 25:1449-1460

81. Kumar H, Lund R, Laiho A et al (2014) Gut microbiota as an epigenetic regulator: pilot study based on whole-genome methylation analysis. MBio 5, e02113

82. Hofmann AF, Hagey LR (2008) Bile acids: chemistry, pathochemistry, biology, pathobiology, and therapeutics. Cell Mol Life Sci 65:2461-2483

83. Trabelsi MS, Daoudi M, Prawitt J et al (2015) Farnesoid X receptor inhibits glucagon-like peptide-1 production by enteroendocrine $\mathrm{L}$ cells. Nat Commun 6:7629

84. Selwyn FP, Csanaky IL, Zhang Y, Klaassen CD (2015) Importance of large intestine in regulating bile acids and glucagon-like peptide1 in germ-free mice. Drug Metab Dispos 43:1544-1556

85. McMahan RH, Wang XX, Cheng LL et al (2013) Bile acid receptor activation modulates hepatic monocyte activity and improves nonalcoholic fatty liver disease. J Biol Chem 288:11761-11770 
86. Wang YD, Chen WD, Yu D, Forman BM, Huang W (2011) The Gprotein-coupled bile acid receptor, Gpbar1 (TGR5), negatively regulates hepatic inflammatory response through antagonizing nuclear factor kappa light-chain enhancer of activated B cells (NF-kappaB) in mice. Hepatology 54:1421-1432

87. Gadaleta RM, van Erpecum KJ, Oldenburg B et al (2011) Farnesoid $\mathrm{X}$ receptor activation inhibits inflammation and preserves the intestinal barrier in inflammatory bowel disease. Gut 60:463-472

88. Islam KB, Fukiya S, Hagio M et al (2011) Bile acid is a host factor that regulates the composition of the cecal microbiota in rats. Gastroenterology 141:1773-1781

89. Everard A, Lazarevic V, Derrien M et al (2011) Responses of gut microbiota and glucose and lipid metabolism to prebiotics in genetic obese and diet-induced leptin-resistant mice. Diabetes 60:27752786

90. Lindsay KL, Brennan L, Kennelly MA et al (2015) Impact of probiotics in women with gestational diabetes mellitus on metabolic health: a randomized controlled trial. Am J Obstet Gynecol 212: 496.e491-411

91. Maurer AD, Eller LK, Hallam MC, Taylor K, Reimer RA (2010) Consumption of diets high in prebiotic fiber or protein during growth influences the response to a high fat and sucrose diet in adulthood in rats. Nutr Metab (Lond) 7:77

92. Rautava S, Collado MC, Salminen S, Isolauri E (2012) Probiotics modulate host-microbe interaction in the placenta and fetal gut: a randomized, double-blind, placebo-controlled trial. Neonatology 102:178-184
93. Luoto R, Laitinen K, Nermes M, Isolauri E (2010) Impact of maternal probiotic-supplemented dietary counselling on pregnancy outcome and prenatal and postnatal growth: a double-blind, placebo-controlled study. Br J Nutr 103:1792-1799

94. Ilmonen J, Isolauri E, Poussa T, Laitinen K (2011) Impact of dietary counselling and probiotic intervention on maternal anthropometric measurements during and after pregnancy: a randomized placebocontrolled trial. Clin Nutr 30:156-164

95. Lindsay KL, Kennelly M, Culliton M et al (2014) Probiotics in obese pregnancy do not reduce maternal fasting glucose: a double-blind, placebo-controlled, randomized trial (Probiotics in Pregnancy Study). Am J Clin Nutr 99:1432-1439

96. Laitinen K, Poussa T, Isolauri E (2009) Probiotics and dietary counselling contribute to glucose regulation during and after pregnancy: a randomised controlled trial. Br J Nutr 101:1679-1687

97. Schultz M, Gottl C, Young RJ, Iwen P, Vanderhoof JA (2004) Administration of oral probiotic bacteria to pregnant women causes temporary infantile colonization. J Pediatr Gastroenterol Nutr 38: 293-297

98. Gueimonde $\mathrm{M}$, Sakata S, Kalliomäki M, Isolauri E, Benno Y, Salminen S (2006) Effect of maternal consumption of Lactobacillus GG on transfer and establishment of fecal bifidobacterial microbiota in neonates. J Pediatr Gastroenterol Nutr 42:166-170

99. Ismail IH, Oppedisano F, Joseph SJ, Boyle RJ, Robins-Browne RM, Tang ML (2012) Prenatal administration of Lactobacillus rhamnosus has no effect on the diversity of the early infant gut microbiota. Pediatr Allergy Immunol 23:255-258 